\title{
La presencia de la arquitectura reformada en el Premio Internazionale Frate Sole (1996-2016)
}

The Presence of Protestant Architecture in the Frate Sole International Prize (1996-2016)

Giorgio Della Longa · Consulta dell'Ufficio Liturgico Nazionale CEl, Roma (Italia)

https://doi.org/10.17979/aarc.2017.5.0.5142

\section{RESUMEN}

Este texto pretende analizar la presencia de la arquitectura protestante en las seis ediciones del Premio Internazionale di Architettura Sacra Frate Sole (1996-2016). Una presencia que se ha ido incrementando con el paso de los años, aunque lograra su máximo reconocimiento ya en la primera edición, cuando el premio recayó en el conjunto de tres capillas construidas por el arquitecto japonés Tadao Ando.

Conviene recordar el carácter específico del Premio Internacional Frate Sole (en cada convocatoria sólo se pueden presentar las iglesias terminadas diez años atrás), así como su vocación inclusiva, contemporánea y universal de promoción de la arquitectura sacra.

Se analizarán las propuestas significativas — más allá de las premiadas — recibidas de las distintas denominaciones protestantes, para incidir en su especificidad. Los resultados obtenidos ayudan a valorar la alta calidad media de las propuestas en el discurso arquitectónico mundial.

\section{PALABRAS CLAVE}

Arquitectura religiosa, arquitectura protestante, Premio Internazionale di Architettura Sacra Frate Sole.

\section{ABSTRACT}

This essay is an analysis of the Protestant participation in the six editions of the Premio Internazionale di Architettura Sacra Frate Sole (International Prize of Church Architecture Frate Sole) 1996-2016. A presence that has been increasing over the years, although it achieved its maximum recognition already in the first edition, when the prize went to the set of three chapels built by the Japanese architect Tadao Ando.

It is worth remembering the specific character of the International Prize - in each edition only the churches built in the previous decade can be submitted - and its peculiar contemporary and universal vocation to promote the Church architecture. The meaningful proposals, besides the rewarded ones, which came from the various Protestant denominations, will be analyzed to contextualize their peculiarities. The results obtained in these years mean to suggest the high average quality of these proposal is within the world architectural discourse.

\section{KEYWORDS}

Religious Architecture, Protestant Architecture, Premio Internazionale di Architettura Sacra Frate Sole. 
Fig. 01. Tadao Ando. Capilla en la montaña Rokko, Kobe (Hjogo, Japón), 1985/86; dibujo dedicado al padre Costantino Ruggeri.

$$
\text { To Padve Constantino Ruggeni }
$$

1

El Premio Internazionale di Architettura Sacra, promovido por la Fondazione Frate Sole de Pavia (Italia), fue intensamente deseado por Costantino Ruggeri, fraile franciscano y artista, fallecido en 2007. La obra del padre Costantino, que firmaba sus obras como Frate Sole, ha recibido amplio reconocimiento en el ámbito del arte y de la arquitectura sacra contemporánea.

La Fondazione tiene entre sus objetivos el de promover la «iglesia construida», para que aparezca reconocida la componente artística y la tensión mística que elevan el espacio a lugar de exaltación espiritual. Con esta convicción la Fondazione ha instituido un premio cuadrienal para señalar al autor de una obra que haya cualificado de manera significativa la arquitectura cristiana contemporánea. El premio se concede a los nuevos edificios religiosos en el ámbito de las confesiones cristianas realizados en todo el mundo.

Con la de 2016, son seis las ediciones del Premio Internazionale celebradas a partir de 1996, con una numerosa participación de arquitectos provenientes del mundo entero; han enviado sus contribuciones más de 450 arquitectos, individualmente o reunidos en grupos.
Éste es el elenco de los vencedores: Tadao Ando (1996), Álvaro Siza (2000), Richard Meier (2004), John Pawson (2008), Cristian Undurraga (2012) y Rafael Moneo en la última edición del Premio (2016).

\section{2}

Hace tiempo que estoy involucrado en la Comisión del Jurado, además de formar parte del Comité Científico de la Fundación. Es de justicia precisar que la síntesis que sigue no es una especie de ranking de méritos, ni mío personal ni, mucho menos, del jurado. Deseo presentar una panorámica que abarque las iglesias cristianas pertenecientes al mundo protestante que se han presentado al concurso (Fondazione Frate Sole 2017).

Hay que decir que el número de proyectos de iglesias protestantes ha ido creciendo con el tiempo; sobre todo en las últimas dos ediciones del Premio, la participación ha sido conspicua y de alta calidad global.

Creo que es justo poner de relieve el hecho de que la calidad difusa que se adivina en las iglesia reformadas - en general, se trata de proyectos de una elevada calidad arquitectónica - supera la calidad media de las iglesias católicas presentadas al concurso. 

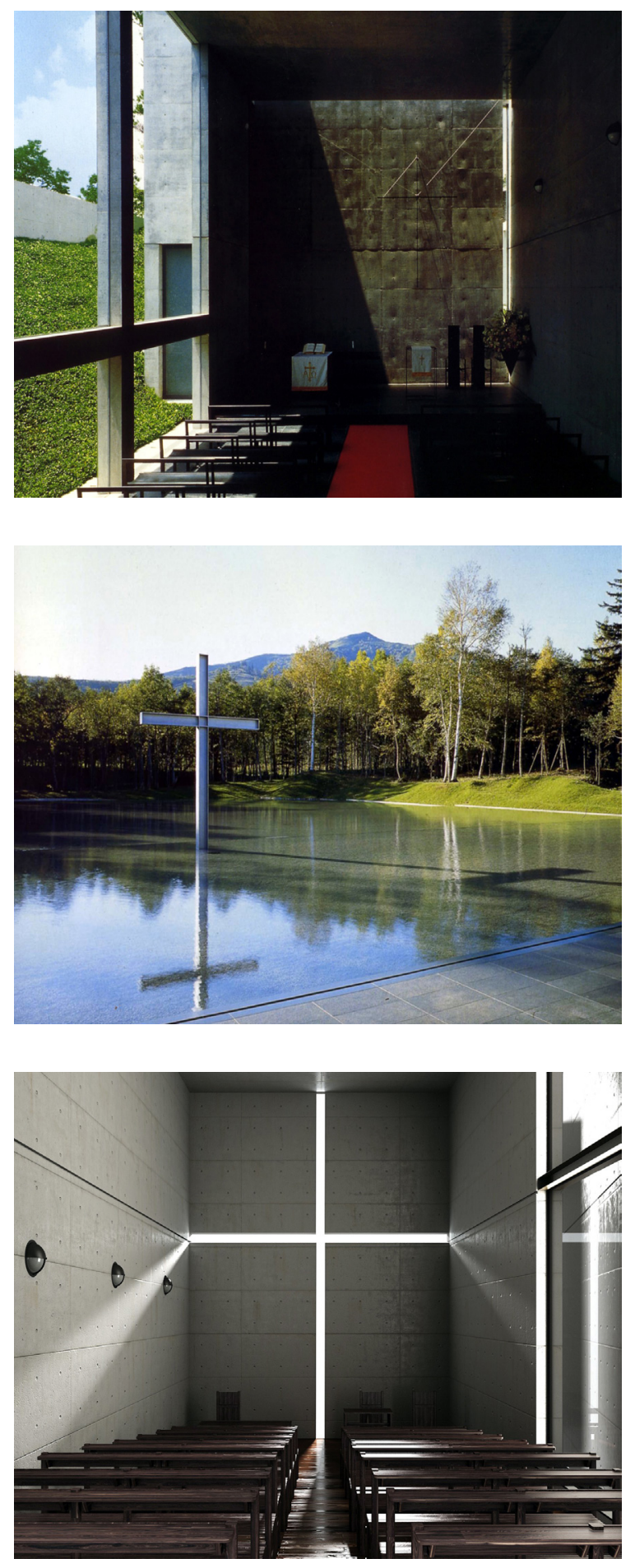

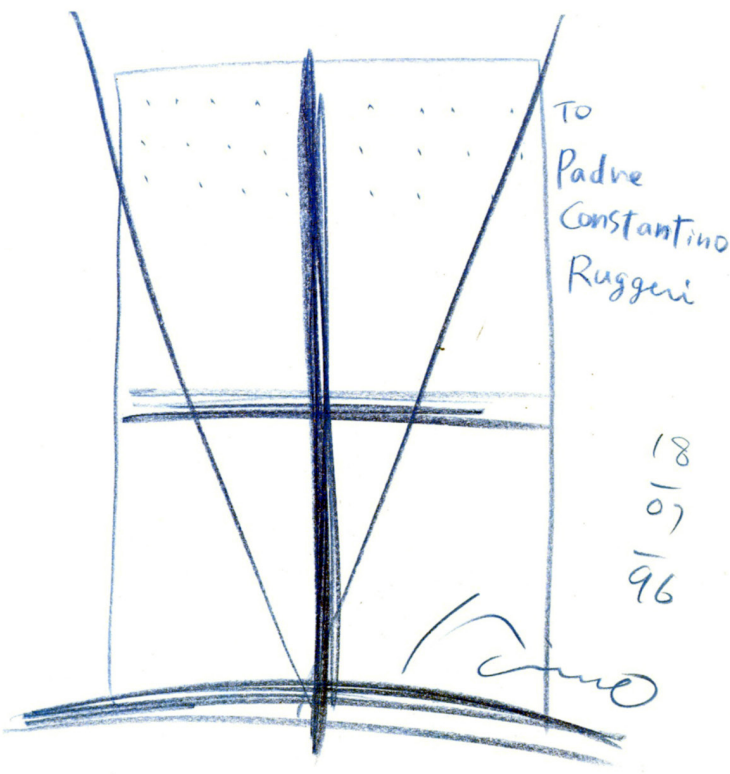

Fig. 02. Tadao Ando. Capilla en la montaña Rokko, Kobe (Hjogo, Japón), 1985/86. Fig. 03. Tadao Ando. Iglesia del agua, Tomamu (Hokkaido, Japón), 1985/88.

Fig. 04. Tadao Ando. Iglesia de la luz, Iberaki (Osaka, Japón), 1987/89.

Fig. 05. Tadao Ando. Iglesia de la luz, Iberaki (Osaka, Japón), 1987/89; dibujo dedicado al padre Costantino Ruggeri. 
3

En la primera edición del Premio, año 1996, a pesar de la supremacía numérica de las iglesias católicas, el arquitecto japonés Tadao Ando es declarado vencedor (Fondazione Frate Sole 1996). Presenta tres pequeñas obras muy célebres: la capilla sobre el monte Rokko, en Kobe-Hjogo (1985/86), la iglesia del Agua en Tomamu-Hokkaido (1985/88) y la iglesia de la Luz en Iberaki-Osaka (1987/89) (Fig. 01-05).

Es interesante, en este caso, tratándose de las arquitecturas premiadas, extrapolar las motivaciones del texto oficial del Jurado:

«La emoción poética que distingue la obra de Tadao Ando, consigue una alta comunicación de la presencia del espíritu de Dios, a través del parsimonioso y sabio uso de la relación entre espacio superficie y luz. Es fuente de significativa emoción también el modo en el cual, en su obra, el paisaje y los elementos naturales - plantas, cielo, agua - se convierten en operadores poéticos en la construcción del lugar sagrado, dilatando así el mensaje arquitectónico hacia un horizonte cósmico. El Premio Internazionale di Architettura Sacra reconoce la validez artística de los espacios sacros de Tadao Ando como ámbitos capaces de aportar verdad visible al acto interior de la oración. Por otra parte, el Premio intenta exprimir una orientación hacia la investigación arquitectónica, para que los principios de la sencillez, el rigor, la pureza y la armonía de las formas sean entendidos como valores primarios insustituibles».

En todas las obras está el tema recurrente de la cruz, en toda su significación simbólica. Como proyección sobre la tierra de encuentro entre el pilar y la viga que comparten la gran apertura del espacio sacro (capilla sobre el monte Rokko): como signo perentorio que se yergue del agua en el espejo artificial que precede a la capilla (iglesia sobre el Agua): como signo luminoso generado por la excavación de la materia en el hormigón armado (iglesia de la Luz).

Concluyo con las palabras del autor, programáticas, referidas a esta última obra: «La iglesia de la Luz representa el esfuerzo realizado por tratar arquitectónicamente y volver —en este sentido - abstracta la luz. El espacio está prácticamente delimitado por

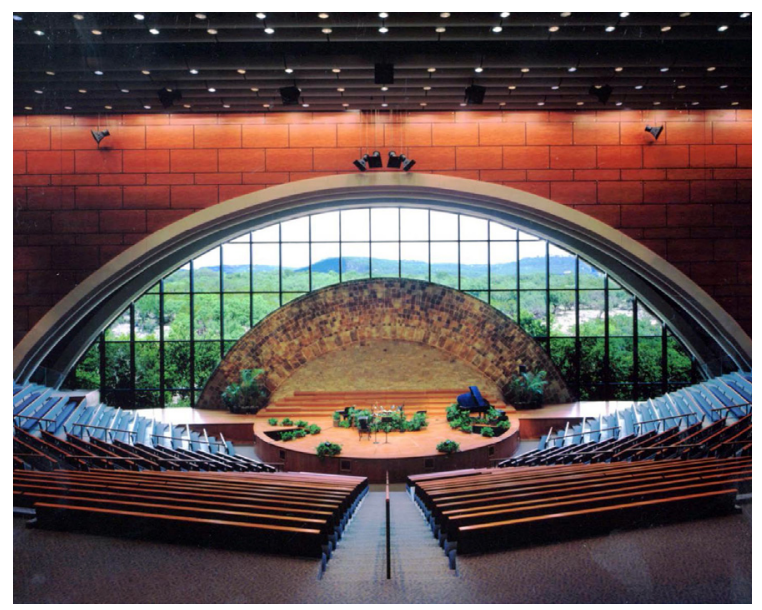

Fig. 06. Overland Partners. Riverbend Church, Austin (Texas, EEUU), 1998.

sólidos muros de hormigón; en el interior se da una profunda oscuridad: en esta oscuridad flota una cruz de luz aislada y no hay nada más. La luz exterior que ha sido manipulada arquitectónicamente y permanece abstracta por las aperturas insertas en los muros, introduce tensión en el espacio y lo sacraliza».

\section{4}

En el 2000 crece la participación numérica de las iglesias de confesión protestante (Fondazione Frate Sole 2000).

Resulta llamativa la dimensión del aula —que no encuentra parangón en otras propuestas presentadas - de la Riverbend Church en Austin (Texas, EEUU), de Overland Partners (Fig. 06); y la dimensión no insólita de los grandes espacios de oración de las comunidades evangélicas en las Américas.

Overland Partners adoptan la planta de teatro griego; una enorme cávea que enfrenta el proscenio con la orquesta. El muro de fondo, verdadera y propia escena, posee una gran apertura semicircular acristalada - aparente evocación de un arco iris - que pone en comunicación el fiel con el paisaje circunstante en la orilla del río Colorado.

A pesar de la adopción de un tipo arcaico, el espacio es adaptable para favorecer también la retrans- 

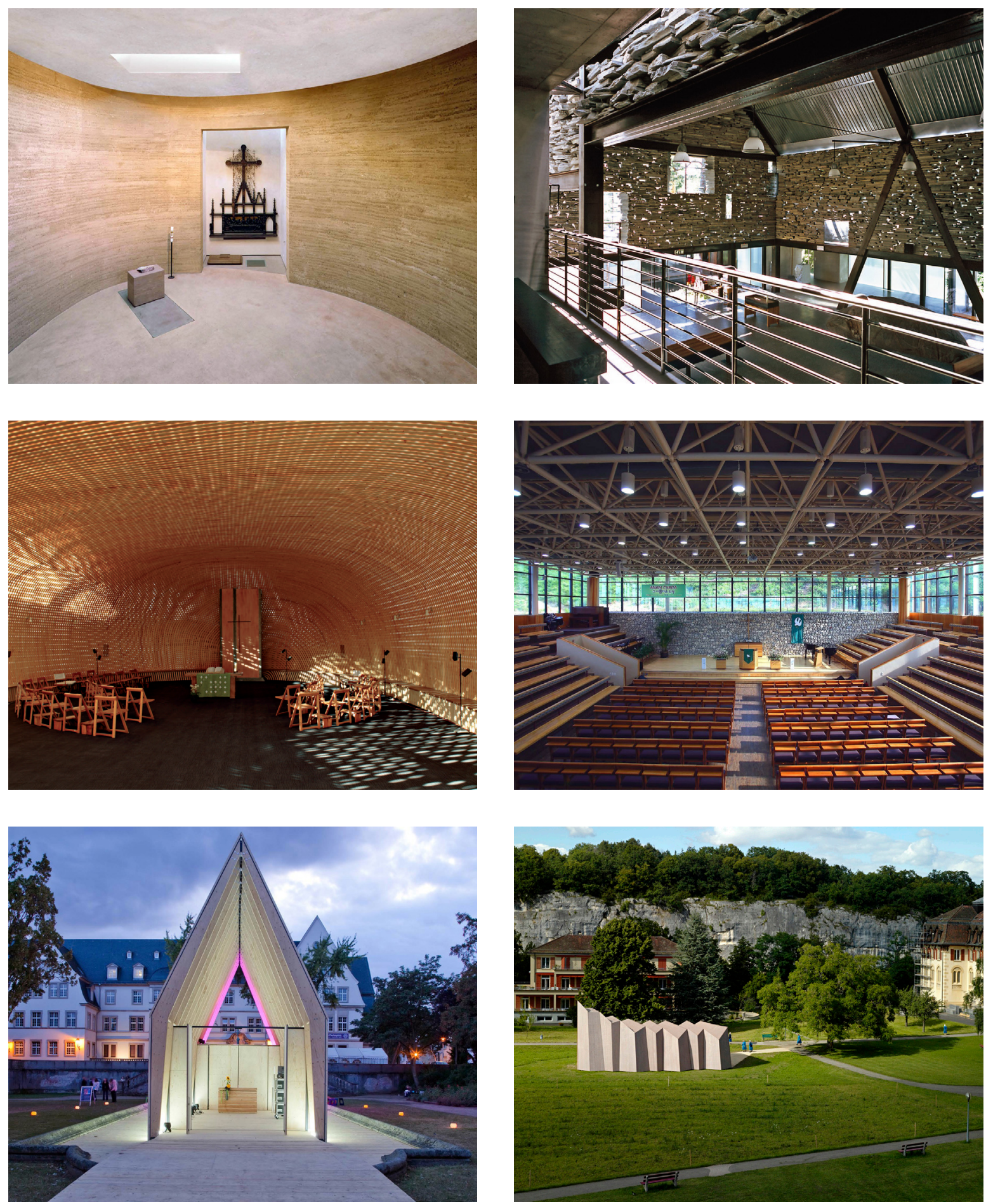
misión televisiva para la múltiple oferta de servicio sacro que la Iglesia de Austin ofrece.

\section{5}

En 2004, entre las pocas obras en concurso se señala la capilla ubicada en Berlín (Alemania), en el área del Memorial del Muro, esto es, la capilla de la Reconciliación de Reitermann y Sassenroth (Fig. 07). Se trata de una construcción muy conocida, también por sus implicaciones de carácter político; surge, de hecho, en lugar de la iglesia protestante cerrada en 1961 y demolida en 1985. La nueva capilla, dedicada en el año 2000, es tanto un lugar de culto protestante como una parte del Memorial del Muro de Berlín. La estructura está constituida por un masivo ovoide de tapial rodeado por una especie de vallado de madera, un diafragma que permite a la luz natural penetrar en el interior y de volverse incorpóreo si se alumbra desde dentro. El espacio de oración está iluminado por una sola linterna colocada sobre la vertical del altar (Fondazione Frate Sole 2004).

\section{6}

Año 2008. Es una edición marcada por algunas intervenciones refinadas que permiten percibir una diversificación muy acentuada entre los usos y las propuestas arquitectónicas (Vaccari 2008).

Entre ellas, la iglesia de Mortensrud, en los alrededores de Oslo (Noruega), obra de Jan Olav Jensen y Børre Skodvin (Fig. 08). El comitente es Den Norske Kirke. Iglesia luterana aue forma parte de

Fig. 07. Rudolf Reitermann y Peter Sassenroth. Capilla de la Reconciliación, Berlín (Alemania), 2000.

Fig. 08. Jan Olav Jensen y Børre Skodvin. Iglesia,

Mortensrud, Oslo (Noruega), 1998/2000.

Fig. 09. Marc Rolinet. Capilla de las Diaconisas de Reuilly, Versalles (Francia), 2006/07.

Fig. 10. Sichoon-Chung. Iglesia Presbiteriana Youngrak, Gumi (Corea del Sur), 2004/05.

Fig. 11. Raum-z Architekten. LichtKirche, capilla móvil de la Iglesia Evangélica de Essen y Nassau (Alemania), 2009. Fig. 12. Localarchitecture y Bureau d'Architecture Danilo Mondada. Capilla temporal para las Diaconisas de St. Loup, Pompaples (Lausana, Suiza), 2008/09. la Comunión de Porvoo, que se significará por otros complejos religiosos en los años siguientes.

Inmersa en la naturaleza, la iglesia, de planta tradicional y con una elocuente cubierta de faldones, dialoga con el ambiente circunstante a través de un uso insólito de los materiales. De hecho, está parcialmente cerrada por un diafragma de lajas de piedra colocadas en seco, por el cual se filtra una abundante luz natural que caracteriza fuertemente el espacio de culto.

La capilla de la Fondation Diaconesses de Reuilly - comunidad religiosa protestante que trabaja en la asistencia sanitaria y social- en Versalles (Francia), es obra de Marc Rolinet (Fig. 09).

La capilla, construida en el interior de un parque, es una estructura en armonía con el ambiente natural y dotada de un carácter propio. La geometría triangular de la envolvente de vidrio transparente engloba una forma orgánica realizada con listones de madera entrelazados. Un diálogo entre la estereométrica fisicidad del contenedor y la mórbida estructura de la capilla.

La Youngrak Presbyterian Church en Gumi (Corea del Sur), obra del arquitecto Sichoon-Chung, es una propuesta de iglesia rural que da respuesta a la petición de la comunidad local. Está resuelta con un sencillo y unitario espacio fuertemente escalonado para favorecer la participación, completamente acristalado en su perímetro y abierto al diálogo con el entorno natural. Como ocurría en la iglesia de Austin, se trata de una explícita referencia tipológica a la arquitectura griega (Fig. 10).

\section{7}

2012 es el año de una numerosa participación de referencias protestantes - cerca de veinte proyectos-, con un alto nivel cualitativo medio (Vaccari 2012). Mejor sacrificar el comentario de las obras singulares para ofrecer una amplia panorámica y testimoniar la extrema diversidad de las propuestas, ya sea en términos de programa como de respuestas arquitectónicas. Y para comprender, también, cómo estas propuestas vinculan transversalmente lugares muy distantes en el mundo.

Encontramos pequeñas construcciones de carácter temporal, como la LichtKirche, primera iglesia 



Fig. 13. Lassila-Hirvilammi Arkkitehdit. Iglesia de Kärsämäki (Finlandia), 1999/2004.

Fig. 14. Taira Nishizawa Architect. Iglesia Sun-pu, Shizuoka (Japón), 2006/08.

Fig. 15. Coop Himmelb(I)au. Iglesia Evangélica, Hainburg/ Donau (Austria), 2008/10.

Fig. 16. JKMM Arkkitehdit. Iglesia Luterana de Viikki, Helsinki (Finlandia), 2002/05.

Fig. 17. Terje Grønmo. Centro Parroquial, Vardåsen (Noruega), 1998/2003. 
móvil de la Iglesia Evangélica de Essen y Nassau (Alemania), icónica estructura provisional proyectada en madera y vidrio para erigirse en ocasión de eventos religiosos de carácter público en la ciudad. El proyecto es de Raum-z Architekten (Fig. 11).

También provisional es la capilla encargada por la Institution des Diaconesses de St. Loup, de Localarchitecture y el Bureau d'Architecture Danilo Mondada (Fig. 12). Realizada completamente en madera laminada, acoge los servicios religiosos de la comunidad durante el periodo de obras de renovación del centro de la comunidad en las Diaconisas de St. Loup en Pompaples, en los alrededores de Lausana (Suiza).

Encontramos construcciones estables, pero siempre de pequeña dimensión, como la refinada propuesta para la iglesia de Kärsämäki (Finlandia), una nueva capilla construida con la técnica de las estructuras de madera del siglo XVIII, en el lugar que ocupaba una iglesia demolida a mediados del siglo XIX. Vencedor de un concurso, el proyecto del equipo Lassila-Hirvilammi arkkitehdit define un espacio cúbico circunscrito por un manto de madera iluminado por una linterna (Fig. 13).

Completamente de madera es también la iglesia Sun-pu, en Shizuoka (Japón), proyectada por Taira Nishizawa Architects para la United Church of Christ in Japan. Una estructura cúbica en madera con un revestimiento interno de pino y una refinada textura de cedro rojo al exterior. También en este caso, un vibrante y diafragmada luz cenital inunda el acogedor espacio interno (Fig. 14).

De pequeña escala es, asimismo, la propuesta de Coop Himmelb(l)au para la iglesia Martin Luther en Hainburg, al este de la capital de Austria. Una iglesia para la comunidad evangélica de Hainburg/Donau, levantada en el lugar de una iglesia desaparecida, articulada según el personal lenguaje de relectura de las formas históricas del famoso grupo de proyectación austríaco (Fig. 15).

Pasemos a los complejos parroquiales; entre estos, merecen destacarse las obras escandinavas como el complejo para la comunidad luterana de Viikki, en las cercanías de Helsinki (Finlandia), del equipo JKMM Architects, donde el aula de la ora- ción asume también el carácter de un espacio cúbico revestido de madera, y en el cual la estructura aparece exhibida y embellecida por la vibración de la luz natural (Fig. 16).

E incluso el complejo parroquial de Vardåsen, en la periferia de Oslo (Noruega), de Terje Grønmo, en el cual, por el contrario, la luz caracteriza el espacio jugando con las magníficas superficies de hormigón armado del aula de la asamblea. Un espacio amplio y luminoso de reunión y oración (Fig. 17).

En el sur de Europa, la Iglesia Evangélica Unida de Tarrasa (Barcelona, España), es la entidad que encarga un complejo que unifique los servicios religiosos y los de carácter social. Autores del proyecto son el equipo OAB, formado por Carlos y Lucía Ferrater. Se trata de una única y rigurosa placa de la cual emergen los distintos volúmenes del programa; una arquitectura caracterizada por un extremo rigor compositivo, por la sobriedad de los materiales y el cuidado por los detalles (Fig. 18).

En los Estados Unidos está situado el Prayer Pavilion of Light, que forma parte del campus de la Phoenix First Assembly de Arizona. Situada en los márgenes de la Phoenix Mountains Preserve y concebido como una «linterna en la cima de la colina», el pabellón de oración está caracterizado por un rigor extremo; diversos muros sostienen un ciborio constituido por dos diafragmas de vidrio. Ciborio que controla la luz natural del día, y de noche se convierte en una cambiante fuente de luz (Fig. 19).

Quisiera, por último, citar dos capillas ecuménicas, obras encargadas por una comunidad de confesión protestante en Finlandia. Las dos muy famosas: la Kamppi Chapel, en el corazón de Helsinki, de K2S Architects, patrocinada por la Helsinki Parish Union y la municipalidad de Helsinki (Fig. 20), y la capilla en Turku de la asociación sin ánimo de lucro St. Henry Chapel — de la cual forman parte siete iglesias cristianas - , obra de Matti y Pirjo Sanaksenaho (Fig. 21). Dos arquitecturas, dos esculturas en el paisaje urbano y periurbano, profundamente diversas en la forma del espacio pero unidas por el uso insistente del mismo material, el pino del norte. 

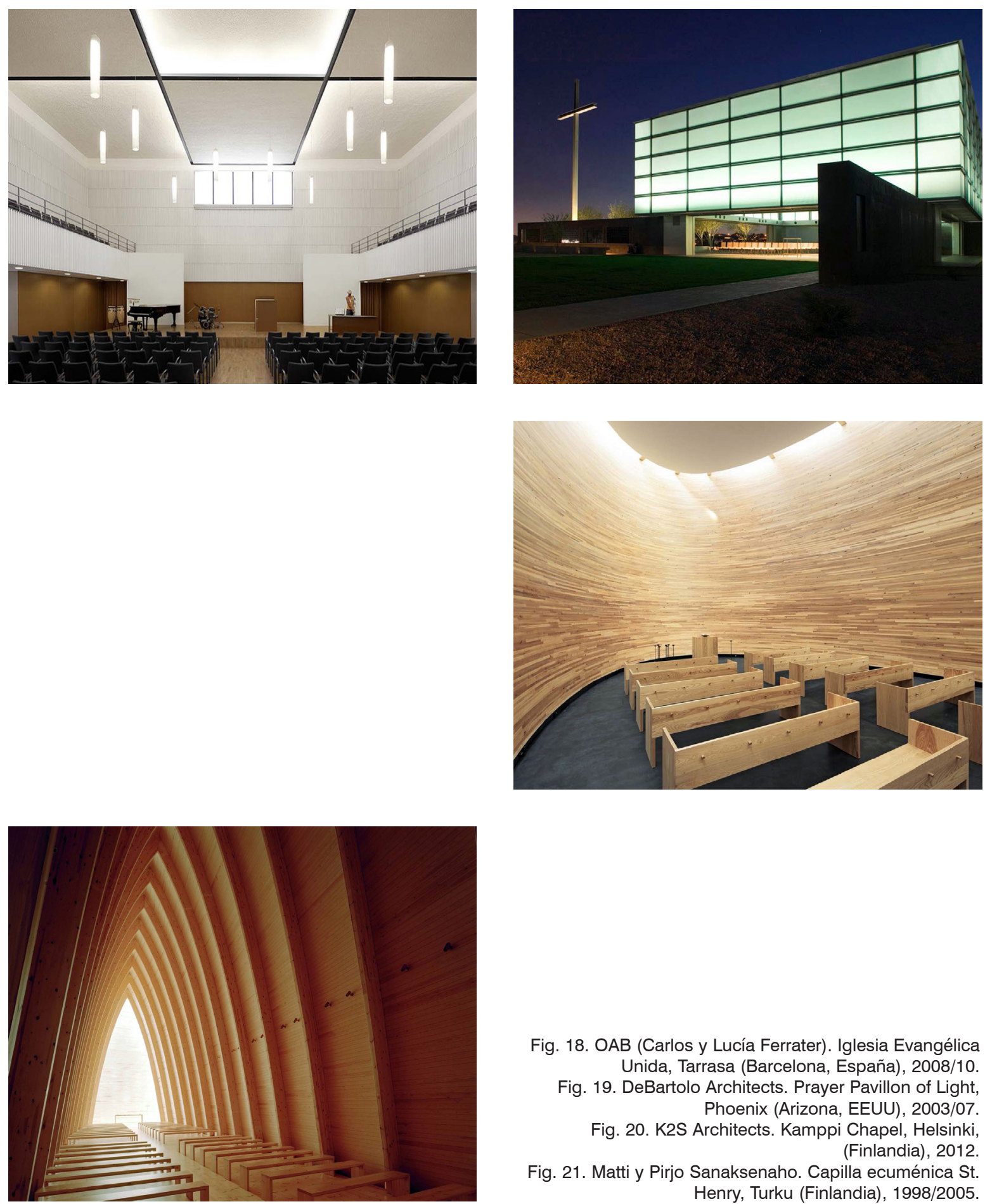

Fig. 18. OAB (Carlos y Lucía Ferrater). Iglesia Evangélica Unida, Tarrasa (Barcelona, España), 2008/10.

Fig. 19. DeBartolo Architects. Prayer Pavillon of Light, Phoenix (Arizona, EEUU), 2003/07.

Fig. 20. K2S Architects. Kamppi Chapel, Helsinki, (Finlandia), 2012.

Fig. 21. Matti y Pirjo Sanaksenaho. Capilla ecuménica St. Henry, Turku (Finlandia), 1998/2005. 

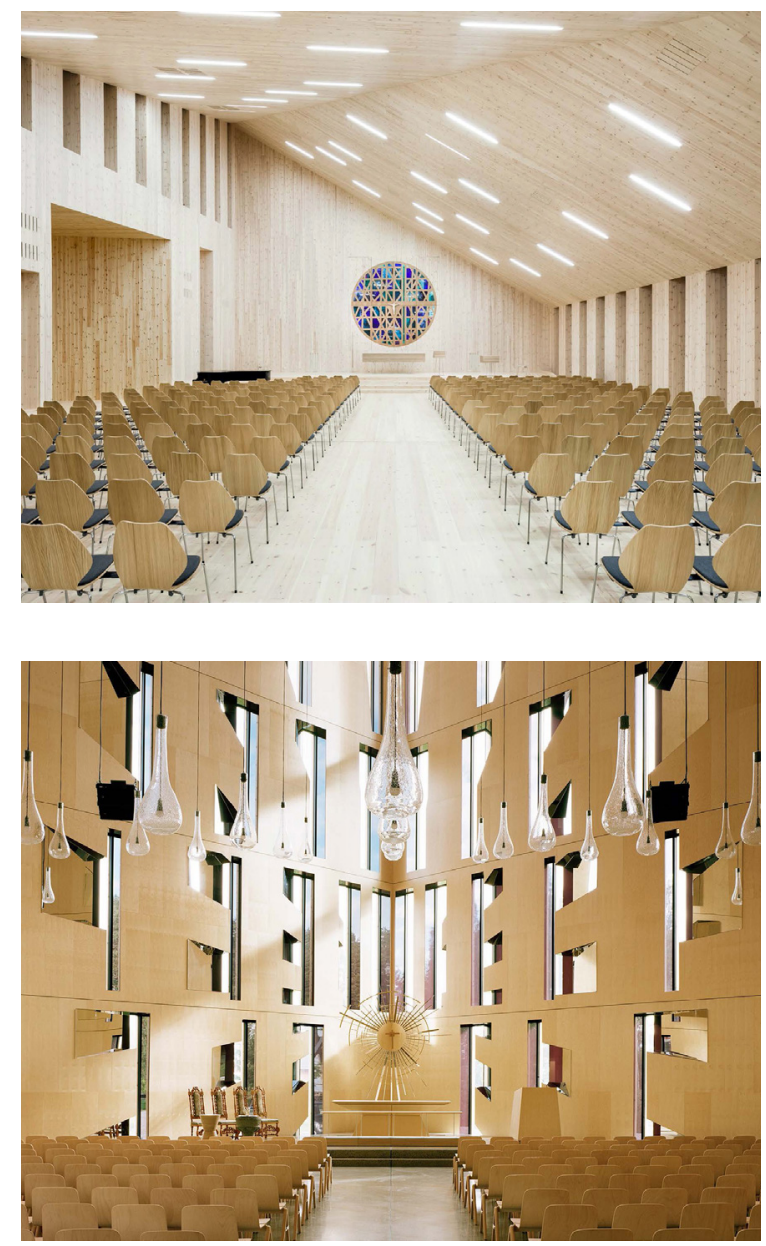

Fig. 22. Reiulf Ramstad Arkitekter. Iglesia, Knarvik, Hordaland (Noruega), 2010/14.

Fig. 23. Espen Surnevik. Iglesia de la comunidad de Våler (Noruega), 2012/15.

\section{8}

Finalmente, en 2016, la presencia de iglesias reformadas también fue numerosa (Vaccari 2016). Me parece interesante detenernos en la arquitectura escandinava para verificar la vitalidad de las propuestas de la Iglesia noruega en particular, que en las varias ediciones se ha distinguido por la calidad de sus intervenciones.

El equipo Reiulf Ramstad Arkitekter es autor de la iglesia para la comunidad de Knarvik, en la costa oriental al norte de Bergen (Noruega), en la cual se yuxtapone la simplicidad de la planta con la nerviosa variedad formal del alzado (Fig. 22).

Espen Surnevik es el autor de la iglesia para la comunidad de Våler, al este de Noruega, construida para sustituir a la iglesia preexistente destruida por un incendio (Fig. 23).

En ambas iglesias domina también la materialidad de la madera que marca las propuestas. En las dos iglesias la forma del edificio religioso se impone como hito en el paisaje, evocando - si bien de diversos modos- el diálogo entre la verticalidad de las iglesias tradicionales y el paisaje nórdico.

En ambos casos - así como en el siguiente y en muchos otros señalados en este escrito-, las obras realizadas son propuestas que han vencido un concurso.

Concluyamos con una intervención significativa de una iglesia con vocación ecuménica. Se trata de una pequeña capilla dentro de una estación de servicio de la autopista A45, que forma parte de la vasta oferta de espacios para el espíritu y la plegaria de las autopistas alemanas.

El comitente es la Autobahnkirche Siegerland. Los autores son Schneider + Schumacher Architekten (Fig. 24).

El exterior es la transposición tridimensional del pictograma que se presenta en las señales de tráfico alemanas para identificar los edificios religiosos; se manifiesta en la autopista con la típica silueta de las iglesias rurales. En el interior presenta un carácter profundamente diverso: el de una acogedora gruta creada por la intersección de paneles de madera laminada en diálogo con el altar y la gran cruz blanca, signo inequívoco de la fe cristiana. 

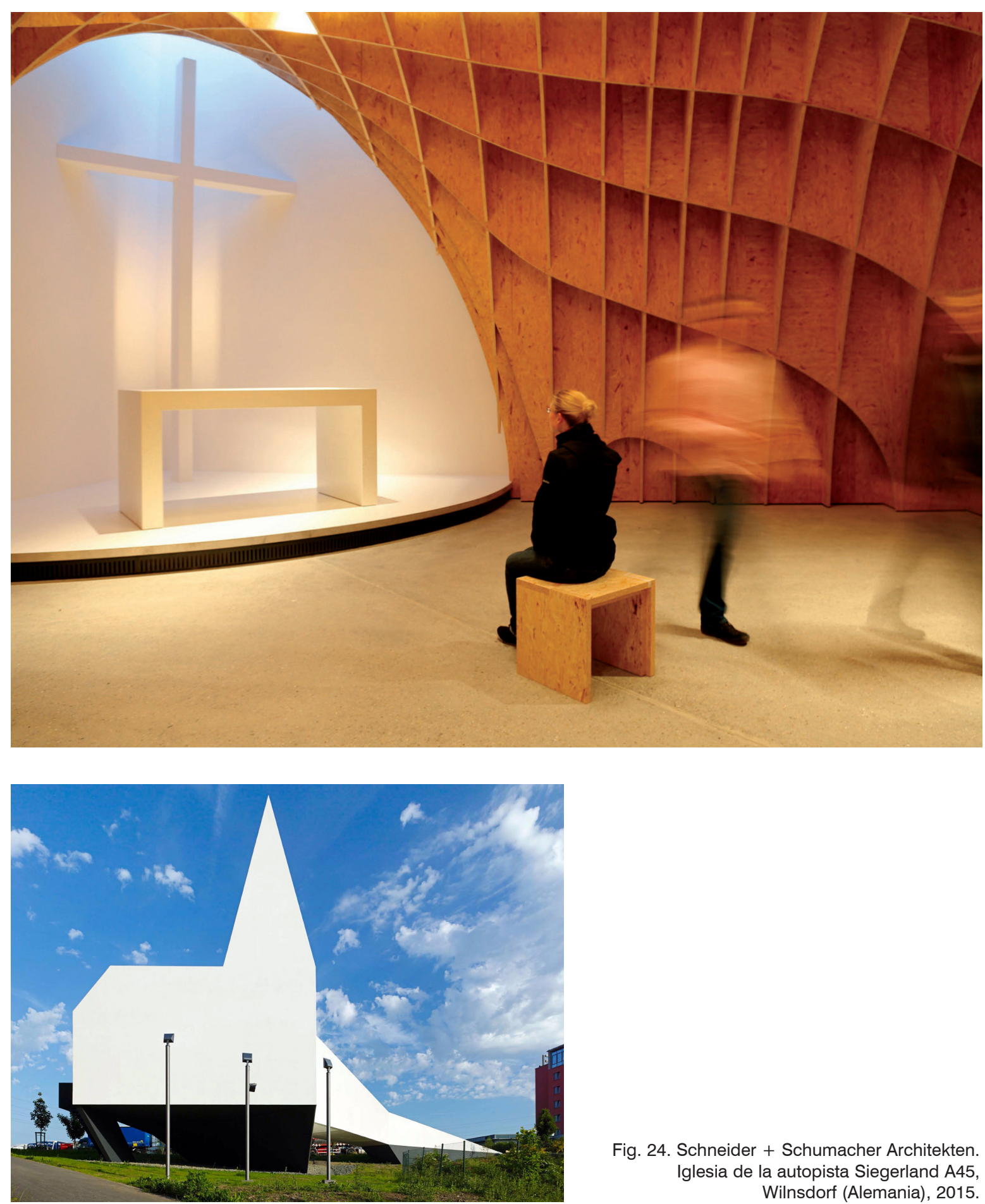

Fig. 24. Schneider + Schumacher Architekten. Iglesia de la autopista Siegerland A45, Wilnsdorf (Alemania), 2015. 


\section{CONCLUSIONES}

En síntesis, creo que se puede confirmar que las obras, de las cuales ha sido presentada aquí una síntesis sustancial, revelan una alta calidad media con puntas de verdadero relieve.

Sólo en algunos casos prevalecen elecciones y lenguajes personales ostentados por los autores; sólo en algunos casos es evidente la dimensión experimental; pero es muy frecuente el recurso a formas basilares, arquetípicas, y al uso de pocos materiales.

Pienso que el uso riguroso de las formas - que en muchos casos recurre al concepto de tipo- es una característica destacada de las realizaciones. Como también el empleo de la materia, a través de la cual se recogen resultados emocionales muy elevados. Por ejemplo, destaca el uso frecuente de la madera, sobre todo para revestir los interiores, que denota una búsqueda de la idea de acogida que, en muchos casos, alude fuertemente a la dimensión doméstica.

En muchos casos, sin embargo, es significativa la utilización de la luz natural como materia primaria de proyecto; no tanto en términos espectaculares como en términos emocionales y simbólicos.

Finalmente, es justo destacar el hecho de que son muchas las iglesias que han sido realizadas como resultado de concursos convocados por las entidades comitentes.

\section{AGRADECIMIENTOS}

Agradezco la ayuda prestada por el arquitecto Andrea Vaccari, de la Fondazione Frate Sole.

\section{BIBLIOGRAFÍA}

«Fondazione Frate Sole». 2017. Con acceso el 1 de agosto de 2017, www.fondazionefratesole.org.

Fondazione Frate Sole. 1996. Fondazione Frate Sole. Tadao Ando. Tre spazi sacri. Torino: Elledici.

Fondazione Frate Sole. 2000. Premio Internazionale di Architettura Sacra «Frate Sole», II edizione. Álvaro Siza. Pavia: Fondazione Frate Sole.

Fondazione Frate Sole. 2004. Premio Internazionale di Architettura Sacra «Frate Sole», III edizione. Richard Meier. Pavia: Fondazione Frate Sole.

Vaccari, Andrea, ed. 2008. Premio Internazionale di Architettura Sacra «Frate Sole», IV edizione. Milán: Skira.

Vaccari, Andrea, ed. 2012. Premio Internazionale di Architettura Sacra «Frate Sole», V edizione. Milán: Skira.

Vaccari, Andrea, ed. 2016. Premio Internazionale di Architettura Sacra «Frate Sole», VI edizione. Pavia: Fondazione Frate Sole.

\section{PROCEDENCIA DE LAS ILUSTRACIONES}

Todas las imágenes pertenecen al archivo de la Fondazione Frate Sole (Pavia, Italia). 